\title{
CHARA Array Adaptive Optics II: Non-Common-Path Correction and Downstream Optics
}

\author{
Theo ten Brummelaar ${ }^{a}$, Xiao Che ${ }^{b}$, Harold McAlister ${ }^{c}$, Michael Ireland $^{d}$, John Monnier $^{b}$, \\ Denis Mourard ${ }^{e}$, Steve Ridgway $^{f}$, Judit Sturmann ${ }^{a}$, Laszlo Sturmann ${ }^{a}$, Nils Turner ${ }^{a}, \&$ \\ Peter Tuthill ${ }^{g}$ \\ ${ }^{a}$ The CHARA Array, P.O. Box 48, Mt Wilson, CA, USA \\ ${ }^{b}$ The University of Michigan, Ann Arbor, MI, USA \\ ${ }^{c}$ CHARA, Georgia State University, Atlanta GA, USA \\ ${ }^{d}$ Australian National University, ACT, Australia \\ ${ }^{e}$ University of Nice, Nice, France

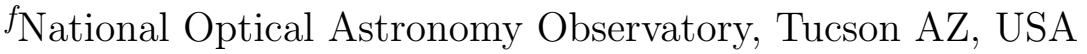 \\ ${ }^{g}$ The University of Sydney, Sydney, NSW, Australia
}

\begin{abstract}
We describe a back-end Adaptive Optics system for the CHARA Array called Lab-AO intended to compensate for non-common path errors between the AO system at the telescopes and the final beam combining area some hundreds of meters away. The system is an on-axis, very small field of view, low order system that will work on star light if enough is present, or will make use of a blue light beacon sent from the telescope towards the laboratory if not enough star light is available. The first of six of these system has been installed and has recently been tested on the sky. Another five will be built for the remaining telescopes later this year.
\end{abstract}

Keywords: Non Common path, Data Reduction, Adaptive Optics, Wavefront Sensor, Deformable Mirror

\section{INTRODUCTION}

The CHARA Array ${ }^{1}$ is an optical/near infrared interferometer consisting of six 1-meter diameter telescopes, the longest baseline of which is 331 meters. With sub-millisecond angular resolution, the CHARA Array is able to spatially resolve nearby stellar systems to reveal their detailed structures. To improve the sensitivity and scientific throughput, the CHARA Array was funded by NSF-ATI for an upgrade of adaptive optics (AO) systems to all six telescopes. The initial grant covers Phase I of the adaptive optics system, which includes an on-telescope Wavefront Sensor ${ }^{2}$ and non-common-path (NCP) error correction. In the long term we hope to correct for atmospheric seeing at the telescope. There are over twenty reflections in each line and some of these are powered elements, while some of them are continually rotating and moving. This means that the NCP problems in an interferometer are much greater than those found in more standard AO systems. We therefore use a beacon and second AO system in the laboratory to correct for these slowly changing NCP errors.

Further author information E-mail: theo@chara-array.org; 


\section{PURPOSE OF ADAPTIVE OPTICS AT THE CHARA ARRAY}

There are several reasons why we want to install full AO systems at the CHARA Array. First, of course, we wish to correct atmospheric seeing, and to this end we are installing full Wave Front Sensors (WFS) at each telescope, more fully described in paper I. ${ }^{2}$ The deformable mirror part of this AO system remains unfunded, and until we have located these funds this WFS will serve as a tiptilt sensor for the active secondary, and will record the wave-fronts arriving at each telescope.

While all AO systems suffer problems from non-common paths in the optics, this is a particularly difficult problem in Interferometry as there are many optical components after the telescopes, and many hundreds of meters of optical path between the telescopes and the beam combiner. We have, therefore, designed and are now installing, a second AO system downstream in the optical laboratory, after the delay lines and beam reducing telescopes, and before the beam combining systems. This system uses the light from a beacon at the telescope, and we call it "Lab-AO".

The Lab-AO system has several aims:

- Correct for quasi-static aberrations, including the rotating aberrations from the telescope. This is the most important function of the Lab-AO system.

- Track the pupil, enabling better pupil alignment throughout the many hundreds of meters of optical path using starlight. Until we had the Lab-AO system we were using paper targets and laser light to align the optics at CHARA. The WFS in Lab-AO give us a much more objective tool for optical alignment.

- Correct for lab seeing and tilt. While not as important as the static aberrations since the delay line building is so large, over $50 \mathrm{~m}$ in length, the seeing in this area can be significant.

- Record atmospheric wavefront errors for use in post-processing. Until we have a full blown AO system at the telescope, we will use the WFS at each telescope to record and time tag the atmospheric seeing above each telescope. This will be used in the post-processing phase and should help us to better calibrate the data.

- Provide focus and pupil position monitoring for system alignment during the day. As shown in Figure 3, there are pupil shear problems in some parts of the optical system. While we have been dealing with these, having an object measuring tool for this sort of problem will be a great improvement.

- Allow a small range of focus adjustment for the beam combiners downstream. With the many hundreds of meters of optical path, five powered elements in the telescopes, delay lines, and beam reducers, as well as powered optics in each of seven beam combiners, defining the focus of each instrument is extremely difficult. We have the ability to add a small amount of focus to the WFS output on Lab-AO so we can make fine adjustments to instrument focus during the night.

- Enable the possibility of open loop AO using the WFS on the telescope. This is the most ambitious part of this program. Until we have DMs at each telescope our only way to correct for atmospheric seeing is to try and use the DM in the laboratory with the telescope WFS in open loop. We will experiment with this until we locate funding for large DMs for each telescope.

\section{OPTICAL LAYOUT}

Since there are already so many optical surfaces in the CHARA layout, it was important for us to find a way of adding a small, relatively inexpensive, DM and WFS to our setup without adding too many more reflections. The final optical layout is shown Figures 1 and 2.

The DM chosen is a 37 actuator Micro-machined Membrane Deformable Mirror (MMDM) built by OKO*, with a $10.5 \mathrm{~mm}$ operational diameter. The optical system already included a Beam Reducing Telescope (BRT) that reduces the $12.5 \mathrm{~cm}$ collimated beam coming from the telescopes, and through the delay lines, down to

\footnotetext{
${ }^{*}$ www.okotech.com
} 


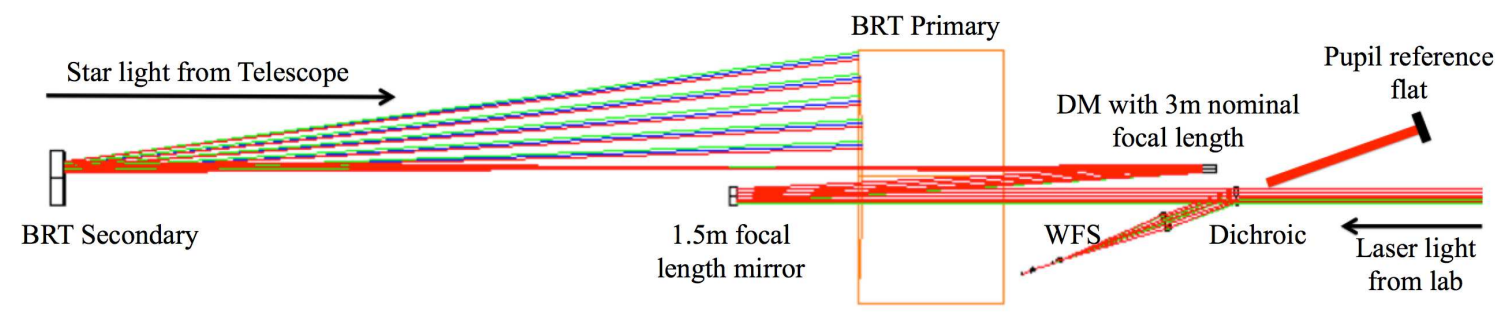

Figure 1. The optical layout of the Laboratory Adaptive Optics System. Starlight enters as a collimated beam from the left, while the alignment and pupil reference laser light enters from the right.

the $2 \mathrm{~cm}$ beam used in the beam combining laboratory. The DM chosen requires that the beam has a spherical default shape, and so the BRT was realigned in order to provide this. The beam is then re-collimated by a small spherical mirror with a $1.5 \mathrm{~m}$ focal length recreating the original $2 \mathrm{~cm}$ beam.

This arrangement conjugates the DM 150m upstream of the BRT which, unfortunately, only matches the telescope pupil for one telescope configuration. Without introducing moving powered optics, we did not find a way to enable us to conjugate the DM to all possible configurations of the Array. Each telescope has a pathlength that is continually changing as we track the fringes and the path-lengths change. An interferometer is an on-axis very small field of view instrument, and so it is not necessary to formally conjugate the telescope pupils, or a specific turbulent layer in the AO system. Furthermore, our models show that this arrangement will work. The DM is, of course, re-imaged on the nearby WFS.

A dichroic is then used to extract the $465 \mathrm{~nm}$ light coming from the beacon at the telescope and send it into the Wave Front Sensor. The alignment laser in the laboratory also reflects off the back of the dichroic. This can then reflect off the pupil reference flat and so reach the WFS providing a pupil reference for the WFS as show in the figure below.

In the WFS a lens re-images the DM into a pupil of $0.95 \mathrm{~mm}$ on an array of micro-lenses with $0.15 \mathrm{~mm}$ pitch and $6.7 \mathrm{~mm}$ focal-length. This gives a \pm 2.3 arcsec field of view per micro-lens. The pixel scale is nominally 0.30 arcsec/pix, with 15 pixels between neighboring images.

\section{CURRENT STATUS AND FIRST RESULTS}

The first of the six Lab-AO was installed in telescope S2 in March of 2014, and was used with the beacon, and trials with starlight, just before this meeting. Two examples are shown in Figure 3.

We have demonstrated that the AO servo loop works well, and that it successfully removes the low order aberrations in the optical system. This installation included computer controlled actuators for the beacon focus and alignment, as well as the ability to correctly co-align the star and beacon light. The current problems are that the beacon is not bright enough, and that the Lab-AO WFS has shown that there is beam shear in the system as currently aligned. The former will be fixed by replacing the LED with a more powerful laser diode, and the latter by a careful re-alignment of the Coude and other parts of the optical path.

The parts for the remaining five Lab-AO systems will be purchased at the beginning of the 2014-2015 financial year and will be installed during our winter engineering break.

We still have a lot to learn about how best to use this system, along with investigating cross calibrating the two WFS and experiment with open loop AO on the sky. Nevertheless, We are confident that our first system has demonstrated that it performs as expected and is able to correct for the quasi-static aberrations, along with the internal laboratory seeing. We hope to use this success to help in our search for funding of the full AO system at the telescopes. 


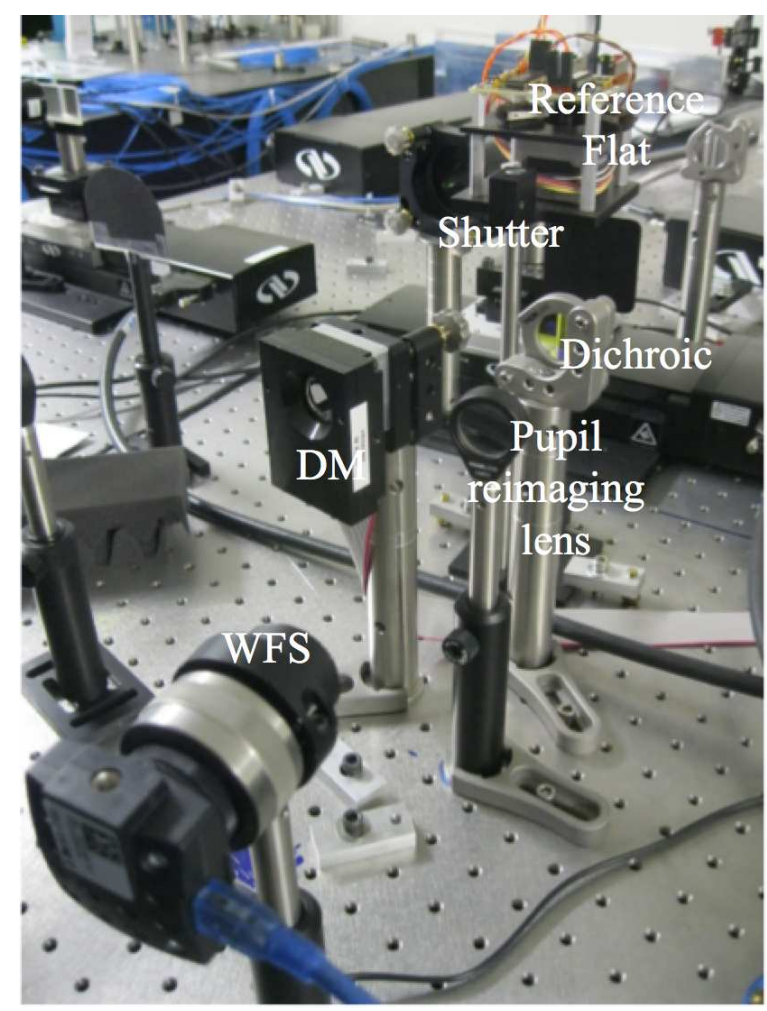

Figure 2. Picture of the Optics installed in the S2 telescope system. This table is in the optics laboratory some hundreds of meters of optical path away from the telescope itself.

\section{ACKNOWLEDGMENTS}

Construction funding for the CHARA Array has been provided by the National Science Foundation through grant AST 94-14449, the W. M. Keck Foundation, the David and Lucile Packard Foundation, and by Georgia State University. The CHARA Phase I AO is funded by the National Science Foundation under Grant AST1106136 Operation of the CHARA Array is funded by the National Science Foundation through NSF grant AST 12-11129 and by Georgia State University through the College of Arts and Sciences.

\section{REFERENCES}

[1] ten Brummelaar et. al., "First Results from the CHARA Array. II. A Description of the Instrument", Ap.J., 628, 453-465 (2005).

[2] Che et. al. "The CHARA Array Adaptive Optics Program I: Common Path Optical and Mechanical Design, and Preliminary on-sky Results", Proc. SPIE, 9146, 15 (2014) 

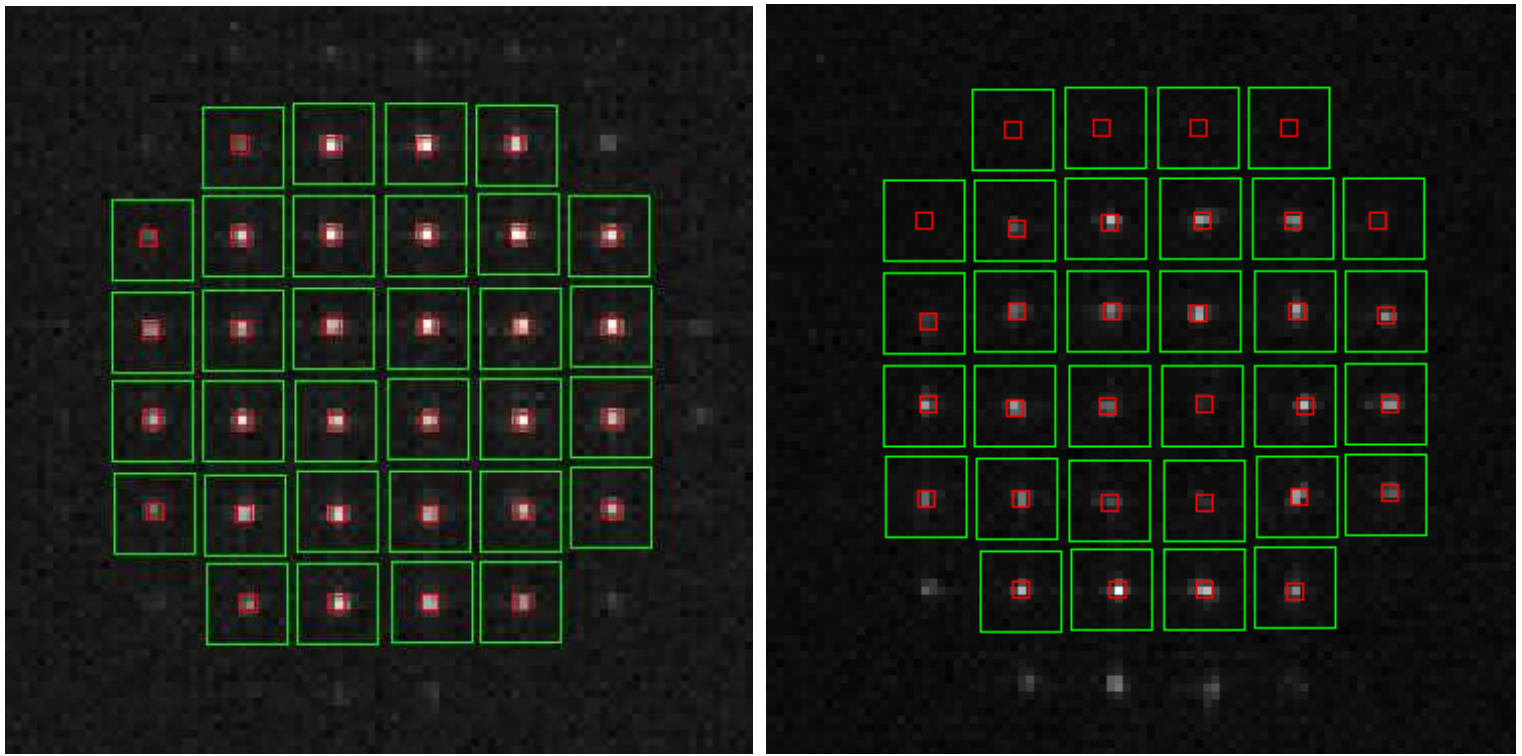

Figure 3. The wave front sensor show all sub-apertures. On the Left we show the system locked onto the laser reference pupil, while on the Right we show the system locked onto the blue beacon on the S2 telescope. Note that some of the top of the beam is missing, and this is thought to be caused by a combination of the small apertures in the delay line and some beam shear somewhere in the system. We are currently working on fixing this problem. Indeed, the WFS is proving to be a very valuable tool for diagnosing this sort of problem. 